\title{
Supplementary Material - Isothermal by design: An accelerated approach to the prediction of the crystallisability of slowly nucleating systems
}

\author{
Peter L. Kaskiewicz', Guangyi Xu', Xiaojun Laił, Nicholas Warren', Kevin J. Roberts ${ }^{\dagger *}$, \\ Colin Morton ${ }^{\S}$, Peter Dowding ${ }^{\S}$, Neil George $e^{¥}$
}

\begin{abstract}
$\dagger^{\dagger}$ School of Chemical and Process Engineering, University of Leeds, Leeds, UK
§Infineum UK Ltd, Milton Hill Business and Technology Centre, Abingdon, UK

${ }^{¥}$ Syngenta UK Ltd, Jealott’s Hill International Research Centre, Berkshire, UK
\end{abstract}

\section{S1. Crystallisation}

Crystallisation occurs in two distinct stages - nucleation and growth. These stages have a large impact on physical and chemical properties of crystals. The crystallisation process route map is given in figure $\mathrm{S} 1$, highlighting the impact of nucleation and growth on these properties. 


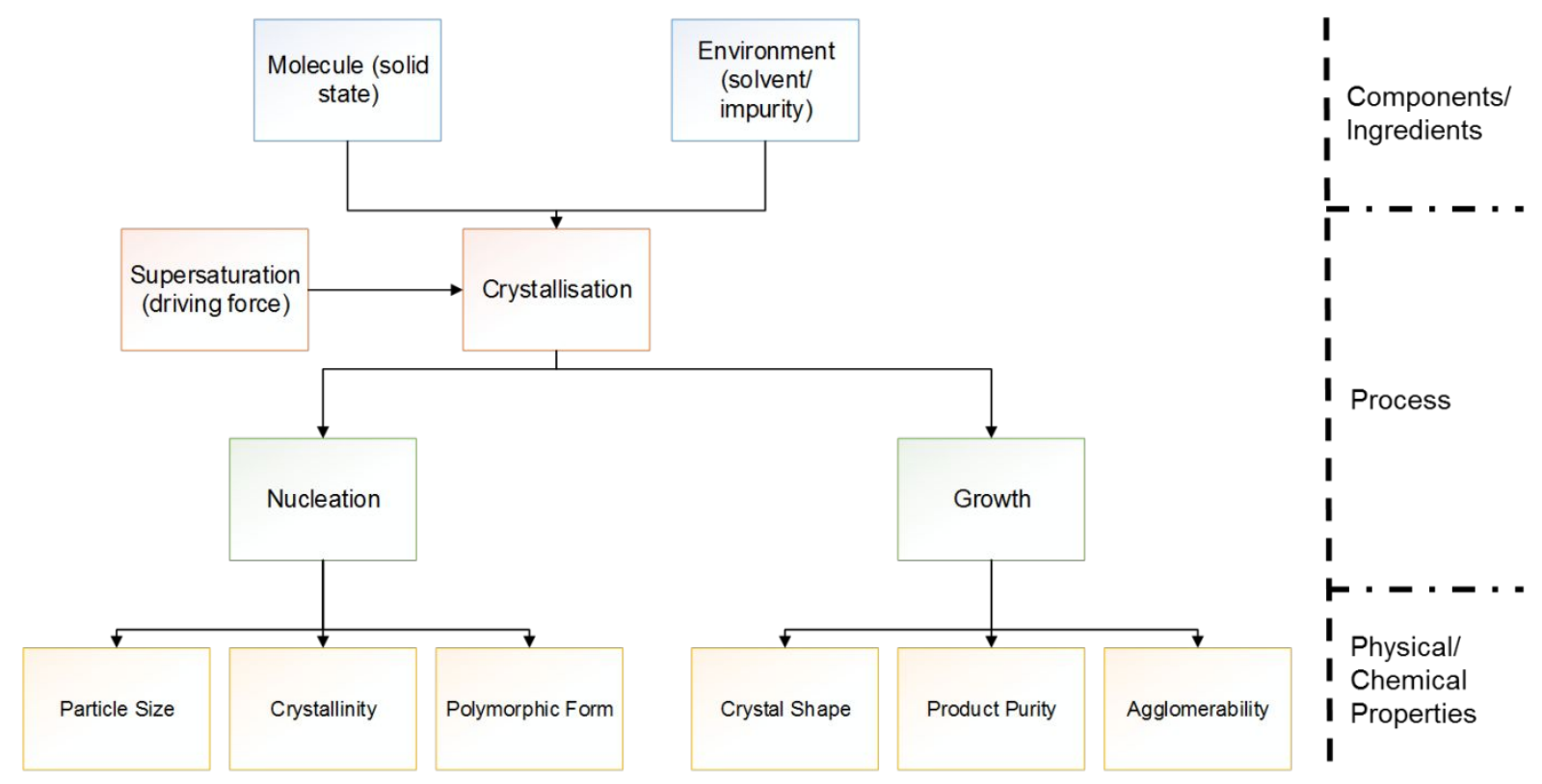

Figure S1. Crystallisation route map schematic. Adapted with permission from Camacho Corzo et al. ${ }^{1}$ Copyright 2017, Springer Science+Business Media B.V.

Nucleation proceeds by either one of two mechanisms: mononuclear or polynuclear nucleation. Polynuclear nucleation occurs when the formation of many nuclei is favourable and can itself be expressed in two different ways, referred to as instantaneous nucleation (IN) and progressive nucleation (PN)2. IN is the process in which all crystal nuclei emerge simultaneously and subsequently grow to form crystals $^{2,3}$. In contrast, in PN crystal nuclei continuously form from solution and subsequently grow in the presence of newly forming nuclei ${ }^{2,4}$ Nucleation can be further characterised by two more categories, primary and secondary nucleation. Primary nucleation describes the process in which nucleation occurs in the absence of any crystalline matter in the solution, whereas secondary nucleation describes the process of nucleation when seed crystals are introduced into the system. Primary nucleation can be further divided into two categories: heterogeneous and homogeneous nucleation. Heterogeneous nucleation (HEN) is caused when sites, usually suspended 
particles or impurities in the solution, provide a surface for nucleation and is usually associated with both instantaneous (IN) and progressive (PN) nucleation, when it is favoured by relatively strong nucleation active sites and relatively weak nucleation active sites, respectively. Homogeneous nucleation (HON) describes spontaneous nucleation and is associated with $\mathrm{PN}^{2}$.

For over half a century nucleation has been described through classical nucleation theory (CNT). This theory proposes a mechanism whereby, in solution, solute molecules come together to form a spherical nucleus and grow to form a macroscopic crystalline structure. It describes the change in Gibbs free energy $(\Delta G)$ associated with the formation of a spherical crystalline nucleus of radius $(r)$ from a supersaturated solution. The overall change in Gibbs free energy due to the formation of a new solid phase from an old phase is a function of the change in surface free energy $\left(\Delta G_{S}\right)$ and volume free energy $\left(\Delta G_{V}\right)^{5}$ and this relationship is expressed as follows:

$$
\begin{gathered}
\Delta G=\Delta G_{S}+\Delta G_{V} \\
\Delta G=4 \pi r^{2} \gamma+\frac{4}{3} \pi r^{3} \Delta G_{v}
\end{gathered}
$$

Where $\gamma$ is the interfacial tension between the crystal and the surrounding supersaturated fluid and $\Delta G_{v}$ is the free energy per unit volume associated with the phase change. 


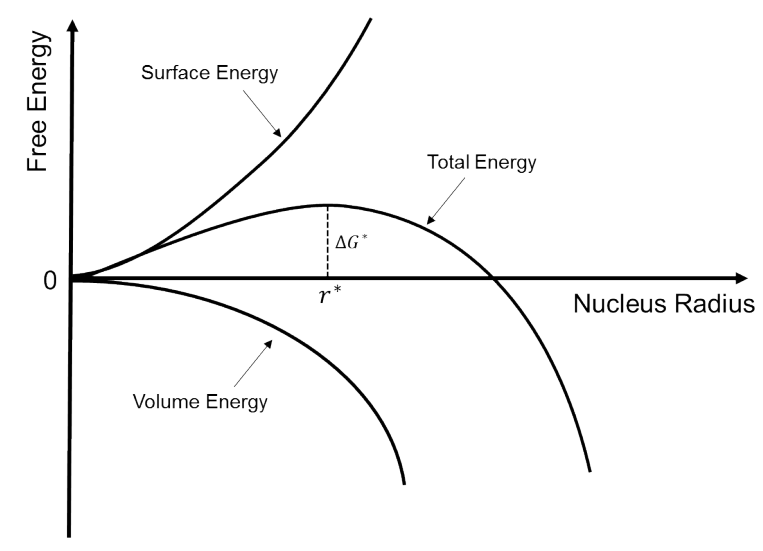

Figure S2. Free energy schematic for nucleation.

Figure S2 demonstrates the relationship between surface and volume terms and illustrates that a critical cluster size must be met before growth is favoured, termed the critical nucleus radius $\left(r^{*}\right)$, with a defined number of molecules in the critical nucleus $\left(i^{*}\right)$. The critical free energy required for this to occur can be described by the equation 5 :

$$
\Delta G^{*}=\frac{16 \pi \gamma^{3} v_{0}^{2}}{3(k T \ln S)^{2}}
$$

Where $v_{0}$ is the volume occupied by a solute molecule in a crystal, $k$ is the Boltzmann constant, $T$ is temperature and $S$ is supersaturation.

Furthermore, it is possible to express the rate at which nucleation occurs $(J)$ in an Arrhenius reaction rate equation, as a function of $\Delta G^{*}: 5$

$$
J=A \exp \left[-\frac{16 \pi \gamma^{3} v_{0}{ }^{2}}{3 k^{3} T^{3}(l n S)^{2}}\right]
$$

Equation S4 indicates that there are three main contributors to the rate of nucleation: temperature, degree of supersaturation and interfacial tension. 


\section{S2. Experimental apparatus}

The Technobis Crystal 16® system ${ }^{6}$, figure S3, was used for all experimental studies. It is made up of sixteen wells, which can hold $1.5 \mathrm{ml}$ disposable glass vials. These are split into four blocks (each has four wells) that are independently temperature controlled to allow different temperature profiles to be set simultaneously, with the use of Peltier elements and an external Julabo bath. The system can be run between -15 ${ }^{\circ} \mathrm{C}$ to $150{ }^{\circ} \mathrm{C}$ and condensation at cooler temperatures is stopped by a dry air purge system. Transmission data is collected by a laser passing through the vials, providing information about the cloud and clear points of up to 16 solutions simultaneously.
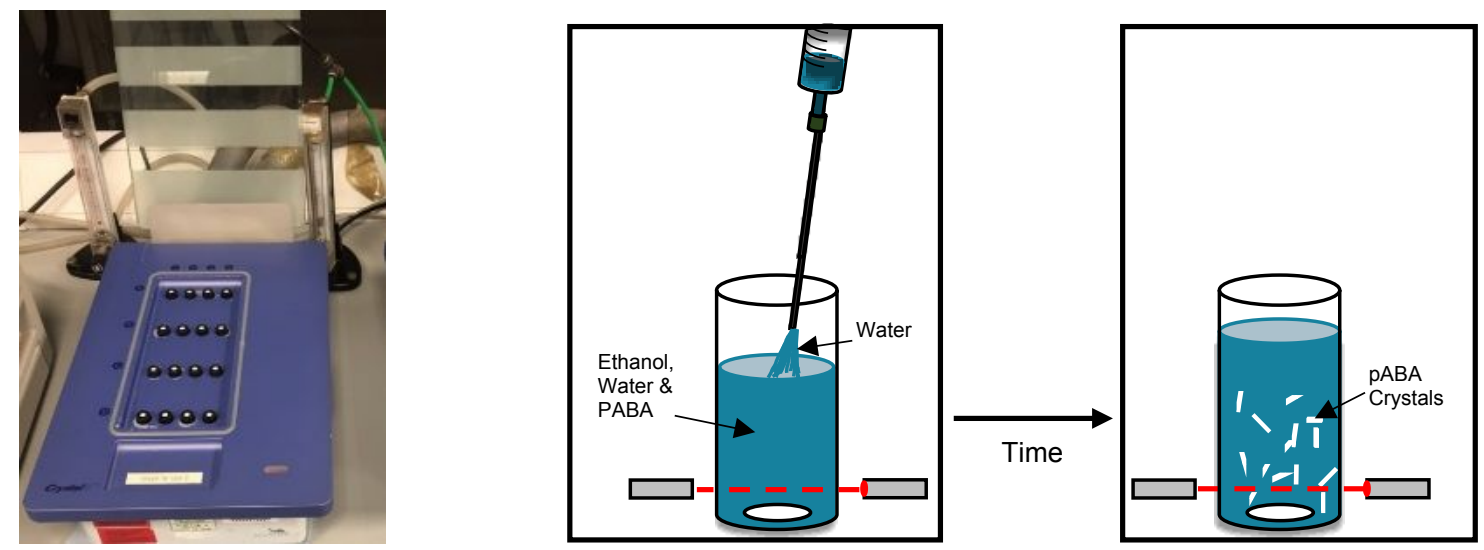

Figure S3. Technobis Crystal 16 system with vials inserted into each well and a schematic of the process undertaken for the $\mathrm{lbD}$ experiments with antisolvent addition to solution vials, inducing crystallisation, as well as transmission detection technique. 


\section{S3. Detailed experimental procedure}

\section{S3.1. Solubility determination}

All solutions were prepared at a minimum of four solution concentrations, with the concentrations used dependent upon the solvent composition (e.g. 140, 160, 180 and $200 \mathrm{~g} \mathrm{~kg}^{-1}$ for pure $\mathrm{EtOH}, 60,90,120$ and $150 \mathrm{~g} \mathrm{~kg}^{-1}$ for $60: 40 \mathrm{wt} \% \mathrm{EtOH}: \mathrm{H}_{2} \mathrm{O}$, and 5, 7, 9 and $11 \mathrm{~g} \mathrm{~kg}^{-1}$ for pure $\mathrm{H}_{2} \mathrm{O}$ ). Eleven solvent compositions ranging in $10 \mathrm{wt} \%$ increments of EtOH from pure $\mathrm{EtOH}$ to pure $\mathrm{H}_{2} \mathrm{O}$ were used for the solubility analysis. pABA, EtOH and $\mathrm{H}_{2} \mathrm{O}$ were weighed into a glass beaker containing a magnetic stirrer bar, on a balance with $\pm 0.1 \mathrm{mg}$ accuracy, with evaporation of solvents limited by covering the beaker with Parafilm. The solutions were subsequently placed on a hot plate and heated to $333 \mathrm{~K}$ at $300 \mathrm{rpm}$ for 1 hour to ensure complete dissolution of pABA and ensure total homogenisation of the solution. $1 \mathrm{ml}$ of solution was then transferred to $1.5 \mathrm{ml}$ disposable glass vials for use in the experimental apparatus, which contained $7 \mathrm{~mm} \times 2 \mathrm{~mm}$ magnetic stirrers, using a Fisherbrand 100-1000 $\mu \mathrm{l}$ micropipette with pre-heated pipette tips $(303 \mathrm{~K})$ to prevent unwanted crystallisation. The $1 \mathrm{ml}$ solutions were subject to different cooling and heating cycles between 333 $\mathrm{K}$ and $278 \mathrm{~K}$ at set rates ( $\mathrm{q}$; where $\mathrm{q}=0.3,0.5,0.7$ and $1 \mathrm{~K} \mathrm{~min}^{-1}$ ), while stirring at 600 rpm.

\section{S3.2. Antisolvent experiments and calorimetry}

All solutions were prepared to ensure a saturation temperature of $293 \mathrm{~K}$ for a mixed solvent solution of 70 wt $\%$ EtOH: 30 wt $\% \mathrm{H}_{2} \mathrm{O} E t O H: \mathrm{H}_{2} \mathrm{O}$ and pABA. The solutions were weighed and prepared in the same way previously described. Solution volumes placed into the glass vials ranged from $0.80 \mathrm{ml}$ to $0.20 \mathrm{ml}$, in $0.05 \mathrm{ml}$ increments. All 
solution volumes were made up to $1 \mathrm{ml}$ after antisolvent addition, meaning, for example, for a vial containing $0.80 \mathrm{ml}$ of solution, $0.20 \mathrm{ml}$ antisolvent was added.

In order to create an IbD approach, calibrations based upon solution calorimetry were performed. For this, a minimum of 5 initial volumes were selected $(0.80 \mathrm{ml}, 0.65$ $\mathrm{ml}, 0.50 \mathrm{ml}, 0.30 \mathrm{ml}$ and $0.20 \mathrm{ml}$ ) for the calibration at a selected temperature (ranging from $293 \mathrm{~K}$ to $301 \mathrm{~K}$ in $2 \mathrm{~K}$ increments), providing enough points for an accurate calibration over the entire range of solution volumes used. Four different antisolvent temperatures were selected, e.g. for a $297 \mathrm{~K}$ isothermal solution temperature, antisolvent temperatures of $293 \mathrm{~K}, 287 \mathrm{~K}, 281 \mathrm{~K}$ and $274 \mathrm{~K}$ were used for the calibrations. Solutions were held at constant temperature, stirring at $900 \mathrm{rpm}$, with the antisolvent held at constant temperature, stirring at $700 \mathrm{rpm}$. The calibrations were performed by placing a digital thermometer into the solution vials and then withdrawing the full antisolvent volume with a $1 \mathrm{ml}$ syringe with attached needle, and transferring the contents into the solution vial rapidly, inducing high mixing. The speed of addition meant that the antisolvent was added almost instantaneously, leading to this procedure being described as 'instant addition'. The temperature of the solution was recorded after each addition by taking the value of the highest recorded temperature on the digital thermometer, within 1 second after antisolvent addition. Each calibration was repeated four times. Plots of selected antisolvent temperatures against the temperature of solutions after antisolvent addition were made and linear regressions were plotted with extrapolations to the desired solution temperature indicating the required antisolvent temperature to maintain solution temperature upon addition. This was then used to determine which antisolvent temperature should be used for each induction time experiment at that temperature. This was subsequently tested to ensure 
that the calibrations were accurate and that the solution temperatures were maintained upon addition of the antisolvent at the determined calibration temperatures. It was observed that deviations in solution temperature upon addition of $\mathrm{H}_{2} \mathrm{O}$, over a range of four repeats, were at most $\pm 0.2 \mathrm{~K}$, with solutions quickly returning to set temperatures in a manner of a few seconds. Therefore, this effect was deemed to be negligible.

\section{S3.3. Isothermal antisolvent measurements}

After the calorimetry calibrations were completed, the $\mathrm{lbD}$ experiments were performed. The same procedure was undertaken as described for the calibrations leading to an 'instant addition' methodology, however, all selected solution volumes were used, antisolvent temperatures were selected based upon the calibrations and no digital thermometer was placed in the vials, ensuring no effect on nucleation by introducing another surface on which to crystallise upon. Sixteen repeats were performed for each initial solution volume used at each selected temperature.

The induction time to nucleation was determined from the collected transmission data, which ranged from $100 \%$ for a homogeneous and $0 \%$ for a completely turbid solution. The crystallisation point was taken when the light transmittance dropped from $100 \%$ to just below $95 \%$ for all measurements, to ensure that a stabilisation period for the transmittance of light had taken place and it was crystallisation that had been detected.

The isothermal transmittance data was used to calculate the time between when the antisolvent was added to the vials and when nucleation had occurred, giving the 
induction time. The point at which antisolvent was added was also determined by the transmission data. For initial solution volumes of $0.5-0.8 \mathrm{ml}$, a sharp decrease in transmission occurred upon antisolvent addition, followed by a rapid increased transmittance after mixing. For initial solution volumes of between $0.3 \mathrm{ml}-0.45 \mathrm{ml}$, the opposite occurred and there was an increase in transmittance associated with antisolvent addition. This was due to the low volume initially in the vials, which were too low to get a regular transmission profile. For these solutions the point of antisolvent addition was taken as the point at which transmission drastically increased. Example transmission plots are provided (figure S4).
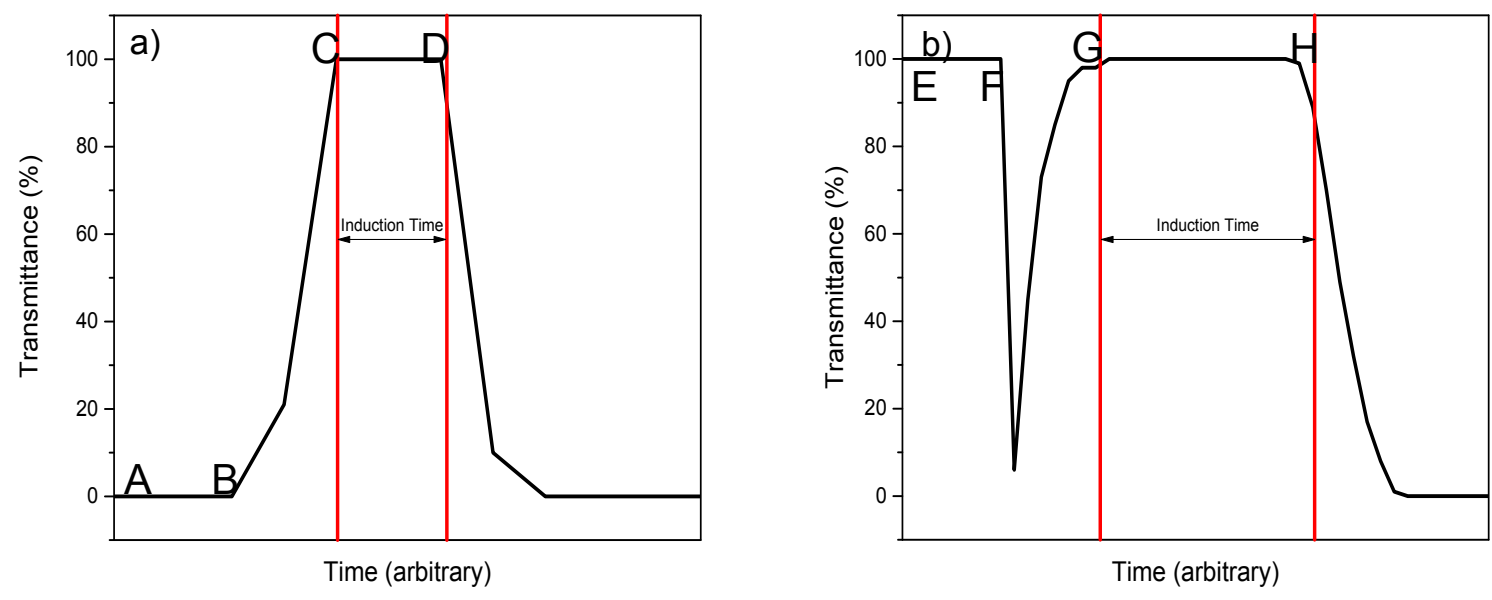

Figure S4. Transmission profiles as a function of time. a) Vial initially containing 0.35 $\mathrm{ml}$ solution. A-B shows transmission profile for undersaturated solution, B indicates time of antisolvent addition, C indicates fully mixed saturated solution, C-D shows transmission profile of supersaturated solution and $D$ indicates onset of crystallisation. b) Vial initially containing $0.55 \mathrm{ml}$ solution. E-F shows transmission profile for undersaturated solution, $F$ indicates time of antisolvent addition, $G$ indicates fully mixed saturated solution, G-H shows transmission profile of supersaturated solution and $\mathrm{H}$ indicates onset of crystallisation. 


\section{S4. Equipment Calibration}

In order to ensure accurate temperature control during isothermal experiments, calibrations were performed on the Technobis Crystal $16 \AA$ system. Four vials containing $1 \mathrm{ml}$ of water were placed in each block, which was programmed to a specific temperature in the range of $18{ }^{\circ} \mathrm{C}$ to $25^{\circ} \mathrm{C}$ and was held at this temperature whilst a thermocouple was used to measure the solvent temperature with $\pm 0.5^{\circ} \mathrm{C}$. The average of the four temperature readings obtained for each block was plotted against the set temperature and fit with a linear regression. The equation of the linear regression was used to ensure that the temperatures of each block could be set to specific values to maintain solutions at desired temperatures. An example calibration performed is shown in figure S5.

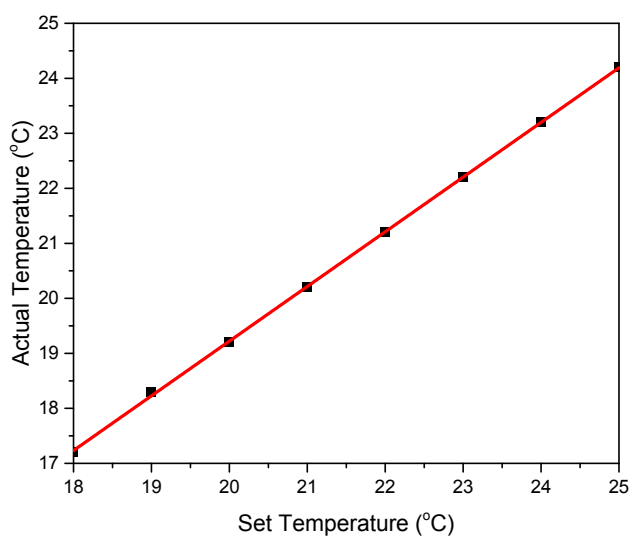

Figure S5. Temperature calibration for the Technobis Crystal $16 \AA$ system. Temperature at which the system was programmed is plotted against the actual temperature of the solvent as measured by a calibrated thermocouple. 


\section{S5. Solubility determination and data}

Table S1 displays the full list of average crystallisation $\left(T_{c}\right)$ and dissolution $\left(T_{\text {diss }}\right)$ temperatures obtained from solubility analysis over the full range of solution concentrations and compositions, alongside the equilibrium saturation temperatures $\left(T_{e}\right)$ calculated from the data. std stands for standard deviation.

\begin{tabular}{|c|c|c|c|c|c|c|c|c|c|c|c|c|c|}
\hline \multicolumn{7}{|c|}{$\mathrm{EtOH}$} & \multicolumn{7}{|c|}{ EtOH: $\mathrm{H}_{2} \mathrm{O} 9: 1$} \\
\hline $\begin{array}{l}\text { Conc. (g } \\
\text { Solvent / } \\
\text { g pABA) }\end{array}$ & $\begin{array}{l}\text { cooling } \\
\text { rate } \\
\left({ }^{\circ} \mathrm{C} / \mathrm{min}\right)\end{array}$ & $T_{c}\left({ }^{\circ} \mathrm{C}\right)$ & std & $T_{\text {diss }}\left({ }^{\circ} \mathrm{C}\right)$ & std & $T_{e}\left({ }^{\circ} \mathrm{C}\right)$ & $\begin{array}{c}\text { Conc. } \\
\text { (g } \\
\text { Solvent } \\
\text { / g } \\
\text { pABA) }\end{array}$ & $\begin{array}{l}\text { cooling } \\
\text { rate } \\
\left({ }^{\circ} \mathrm{C} / \mathrm{min}\right)\end{array}$ & $T_{c}\left({ }^{\circ} \mathrm{C}\right)$ & std & $T_{\text {diss }}\left({ }^{\circ} \mathrm{C}\right)$ & std & $T_{e}\left({ }^{\circ} \mathrm{C}\right)$ \\
\hline \multirow[t]{4}{*}{0.14} & 0.3 & 6.5 & 2.2 & 20.9 & 0.1 & 20.0 & 0.14 & 0.3 & 7.3 & 2.0 & 17.5 & 0.9 & 16.7 \\
\hline & 0.5 & 5.0 & 3.6 & 22.6 & 0.2 & & & 0.5 & 6.4 & 0.7 & 18.0 & 0.8 & \\
\hline & 0.7 & 2.5 & 1.8 & 21.5 & 0.2 & & & 0.7 & 5.5 & 0.0 & 18.1 & 0.1 & \\
\hline & 1.0 & 0.8 & 3.5 & 23.9 & 0.2 & & & 1.0 & 4.8 & 0.2 & 19.2 & 1.1 & \\
\hline \multirow[t]{4}{*}{0.16} & 0.3 & 15.2 & 1.7 & 29.3 & 0.1 & 28.4 & 0.18 & 0.3 & 19.6 & 4.4 & 30.7 & 0.7 & 30.4 \\
\hline & 0.5 & 12.4 & 4.5 & 30.9 & 0.2 & & & 0.5 & 16.5 & 0.2 & 30.5 & 0.7 & \\
\hline & 0.7 & 8.7 & 2.7 & 30.1 & 0.1 & & & 0.7 & 14.4 & 0.6 & 30.9 & 0.8 & \\
\hline & 1.0 & 7.0 & 4.7 & 32.1 & 0.3 & & & 1.0 & 12.1 & 0.2 & 31.0 & 0.8 & \\
\hline \multirow[t]{4}{*}{0.18} & 0.3 & 34.2 & 0.5 & 45.8 & 0.1 & 45.0 & 0.22 & 0.3 & 30.4 & 4.3 & 39.1 & 2.3 & 38.7 \\
\hline & 0.5 & 32.0 & 1.5 & 47.5 & 0.2 & & & 0.5 & 27.0 & 3.3 & 40.2 & 1.0 & \\
\hline & 0.7 & 29.7 & 1.9 & 46.4 & 0.4 & & & 0.7 & 25.6 & 2.5 & 40.9 & 0.7 & \\
\hline & 1.0 & 27.7 & 7.0 & 48.5 & 0.2 & & & 1.0 & 21.4 & 4.6 & 41.0 & 0.8 & \\
\hline \multirow[t]{4}{*}{0.20} & 0.3 & 44.3 & 1.6 & 51.8 & 1.4 & 51.1 & 0.26 & 0.3 & 39.0 & 3.4 & 48.8 & 0.5 & 48.3 \\
\hline & 0.5 & 39.0 & 2.3 & 53.5 & 0.1 & & & 0.5 & 36.1 & 2.6 & 48.8 & 0.5 & \\
\hline & 0.7 & 35.1 & 3.2 & 52.2 & 0.1 & & & 0.7 & 33.0 & 3.1 & 49.5 & 1.2 & \\
\hline & 1.0 & 31.0 & 1.4 & 54.4 & 0.1 & & & 1.0 & 28.5 & 9.4 & 49.6 & 1.2 & \\
\hline
\end{tabular}




\begin{tabular}{|c|c|c|c|c|c|c|c|c|c|c|c|c|c|}
\hline \multicolumn{7}{|c|}{ EtOH: $\mathrm{H}_{2} \mathrm{O} 8: 2$} & \multicolumn{7}{|c|}{ EtOH: $\mathrm{H}_{2} \mathrm{O} 7: 3$} \\
\hline $\begin{array}{l}\text { Conc. (g } \\
\text { Solvent / } \\
\text { g pABA) }\end{array}$ & $\begin{array}{l}\text { cooling } \\
\text { rate } \\
\left({ }^{\circ} \mathrm{C} / \mathrm{min}\right)\end{array}$ & $T_{c}\left({ }^{\circ} \mathrm{C}\right)$ & std & $T_{\text {diss }}\left({ }^{\circ} \mathrm{C}\right)$ & std & $T_{e}\left({ }^{\circ} \mathrm{C}\right)$ & $\begin{array}{c}\text { Conc. } \\
\text { (g } \\
\text { Solvent } \\
\text { / g } \\
\text { pABA) }\end{array}$ & $\begin{array}{l}\text { cooling } \\
\text { rate } \\
\left({ }^{\circ} \mathrm{C} / \mathrm{min}\right)\end{array}$ & $T_{c}\left({ }^{\circ} \mathrm{C}\right)$ & std & $T_{\text {diss }}\left({ }^{\circ} \mathrm{C}\right)$ & std & $T_{e}\left({ }^{\circ} \mathrm{C}\right)$ \\
\hline \multirow[t]{4}{*}{0.14} & 0.3 & 8.8 & 1.1 & 15.2 & 0.2 & \multirow[t]{4}{*}{15.2} & \multirow[t]{4}{*}{0.16} & 0.3 & 16.9 & 2.7 & 26.8 & 0.2 & \multirow[t]{4}{*}{26.0} \\
\hline & 0.5 & 6.6 & 0.8 & 16.3 & 0.1 & & & 0.5 & 15.5 & 2.2 & 26.9 & 0.2 & \\
\hline & 0.7 & 5.6 & 1.0 & 16.3 & 0.2 & & & 0.7 & 13.9 & 1.9 & 27.5 & 0.2 & \\
\hline & 1.0 & 4.9 & 0.0 & 16.4 & 0.1 & & & 1.0 & 12.6 & 1.6 & 28.3 & 0.2 & \\
\hline \multirow[t]{4}{*}{0.18} & 0.3 & 19.4 & 2.5 & 27.5 & 0.2 & \multirow[t]{4}{*}{27.1} & \multirow[t]{4}{*}{0.20} & 0.3 & 26.3 & 1.9 & 35.7 & 0.1 & \multirow[t]{4}{*}{35.0} \\
\hline & 0.5 & 16.1 & 4.3 & 28.1 & 0.1 & & & 0.5 & 25.0 & 1.8 & 35.8 & 0.1 & \\
\hline & 0.7 & 13.8 & 4.1 & 28.5 & 0.1 & & & 0.7 & 24.0 & 1.9 & 36.2 & 0.2 & \\
\hline & 1.0 & 10.0 & 0.9 & 28.6 & 0.0 & & & 1.0 & 23.2 & 2.6 & 36.9 & 0.2 & \\
\hline \multirow[t]{4}{*}{0.22} & 0.3 & 28.1 & 2.8 & 36.4 & 0.2 & \multirow[t]{4}{*}{36.2} & \multirow[t]{4}{*}{0.24} & 0.3 & 34.8 & 1.8 & 42.8 & 0.2 & \multirow[t]{4}{*}{42.1} \\
\hline & 0.5 & 25.7 & 4.7 & 37.0 & 0.1 & & & 0.5 & 31.3 & 2.3 & 42.8 & 0.2 & \\
\hline & 0.7 & 23.5 & 2.6 & 37.5 & 0.2 & & & 0.7 & 27.5 & 1.2 & 44.2 & 1.4 & \\
\hline & 1.0 & 21.0 & 3.5 & 37.5 & 0.1 & & & 1.0 & 24.0 & 1.0 & 44.0 & 0.1 & \\
\hline \multirow[t]{4}{*}{0.26} & 0.3 & 36.1 & 1.9 & 43.9 & 0.2 & \multirow[t]{4}{*}{43.4} & \multirow[t]{4}{*}{0.28} & 0.3 & 41.3 & 0.3 & 49.1 & 0.3 & \multirow[t]{4}{*}{48.5} \\
\hline & 0.5 & 33.5 & 2.3 & 43.8 & 0.3 & & & 0.5 & 40.0 & 0.2 & 49.3 & 0.2 & \\
\hline & 0.7 & 28.3 & 4.5 & 45.2 & 1.3 & & & 0.7 & 39.0 & 0.1 & 49.8 & 0.1 & \\
\hline & 1.0 & 25.5 & 2.2 & 44.8 & 0.0 & & & 1.0 & 38.0 & 0.1 & 50.3 & 0.1 & \\
\hline \multicolumn{7}{|c|}{$\mathrm{EtOH}: \mathrm{H}_{2} \mathrm{O} 6: 4$} & \multicolumn{7}{|c|}{ EtOH: $\mathrm{H}_{2} \mathrm{O} 5: 5$} \\
\hline $\begin{array}{l}\text { Conc. (g } \\
\text { Solvent / } \\
\text { g pABA) }\end{array}$ & $\begin{array}{l}\text { cooling } \\
\text { rate } \\
\left({ }^{\circ} \mathrm{C} / \mathrm{min}\right)\end{array}$ & $T_{c}\left({ }^{\circ} \mathrm{C}\right)$ & std & $T_{\text {diss }}\left({ }^{\circ} \mathrm{C}\right)$ & std & $T_{e}\left({ }^{\circ} \mathrm{C}\right)$ & $\begin{array}{c}\text { Conc. } \\
\text { (g } \\
\text { Solvent } \\
/ g \\
\text { pABA) }\end{array}$ & $\begin{array}{l}\text { cooling } \\
\text { rate } \\
\left({ }^{\circ} \mathrm{C} / \mathrm{min}\right)\end{array}$ & $T_{c}\left({ }^{\circ} \mathrm{C}\right)$ & std & $T_{\text {diss }}\left({ }^{\circ} \mathrm{C}\right)$ & std & $T_{e}\left({ }^{\circ} \mathrm{C}\right)$ \\
\hline \multirow[t]{3}{*}{0.12} & 0.3 & 20.7 & 1.5 & 29.0 & 0.0 & \multirow[t]{3}{*}{22.0} & \multirow[t]{3}{*}{0.10} & 0.3 & 13.3 & 2.4 & 28.1 & 0.1 & \multirow[t]{3}{*}{27.4} \\
\hline & 0.5 & 17.9 & 2.8 & 28.9 & 0.1 & & & 0.5 & 10.4 & 1.0 & 28.5 & 0.1 & \\
\hline & 0.7 & 15.2 & 4.4 & 28.7 & 0.1 & & & 0.7 & 9.3 & 1.4 & 28.4 & 0.2 & \\
\hline
\end{tabular}




\begin{tabular}{|c|c|c|c|c|c|c|c|c|c|c|c|c|c|}
\hline & 1.0 & 12.5 & 1.5 & 29.9 & 0.3 & & & 1.0 & 8.3 & 1.1 & 29.6 & 0.2 & \\
\hline \multirow[t]{4}{*}{0.16} & 0.3 & 31.5 & 4.2 & 37.4 & 0.1 & \multirow[t]{4}{*}{32.1} & \multirow[t]{4}{*}{0.14} & 0.3 & 29.1 & 2.4 & 38.7 & 0.2 & \multirow[t]{4}{*}{38.1} \\
\hline & 0.5 & 29.0 & 1.4 & 37.6 & 0.2 & & & 0.5 & 27.5 & 1.1 & 38.8 & 0.1 & \\
\hline & 0.7 & 26.8 & 4.2 & 36.7 & 4.3 & & & 0.7 & 26.7 & 0.2 & 39.2 & 0.2 & \\
\hline & 1.0 & 25.5 & 0.8 & 38.4 & 0.1 & & & 1.0 & 25.8 & 0.4 & 39.8 & 0.2 & \\
\hline \multirow[t]{4}{*}{0.20} & 0.3 & 38.7 & 1.2 & 45.2 & 0.1 & \multirow[t]{4}{*}{40.5} & \multirow[t]{4}{*}{0.18} & 0.3 & 37.1 & 1.2 & 47.5 & 0.2 & \multirow[t]{4}{*}{48.9} \\
\hline & 0.5 & 36.0 & 2.6 & 45.2 & 0.1 & & & 0.5 & 35.4 & 3.1 & 47.4 & 0.1 & \\
\hline & 0.7 & 34.4 & 1.7 & 45.4 & 0.1 & & & 0.7 & 33.7 & 2.4 & 47.9 & 0.3 & \\
\hline & 1.0 & 31.7 & 2.2 & 46.1 & 0.2 & & & 1.0 & 32.4 & 4.5 & 48.4 & 0.1 & \\
\hline \multirow[t]{4}{*}{0.24} & 0.3 & 43.0 & 1.5 & 50.9 & 0.2 & \multirow[t]{4}{*}{47.5} & \multirow[t]{4}{*}{0.20} & 0.3 & 44.2 & 0.9 & 54.4 & 0.1 & \multirow[t]{4}{*}{53.4} \\
\hline & 0.5 & 38.0 & 2.1 & 51.1 & 0.2 & & & 0.5 & 42.1 & 1.0 & 54.5 & 0.2 & \\
\hline & 0.7 & 33.0 & 4.9 & 49.6 & 0.2 & & & 0.7 & 38.7 & 2.1 & 54.5 & 0.3 & \\
\hline & 1.0 & 29.0 & 4.9 & 52.0 & 0.1 & & & 1.0 & 35.3 & 1.7 & 56.1 & 0.0 & \\
\hline \multicolumn{7}{|c|}{ EtOH: $\mathrm{H}_{2} \mathrm{O} 4: 6$} & \multicolumn{7}{|c|}{ EtOH: $\mathrm{H}_{2} \mathrm{O} 3: 7$} \\
\hline $\begin{array}{l}\text { Conc. (g } \\
\text { Solvent / } \\
\text { g pABA) }\end{array}$ & $\begin{array}{l}\text { cooling } \\
\text { rate } \\
\left({ }^{\circ} \mathrm{C} / \mathrm{min}\right)\end{array}$ & $T_{c}\left({ }^{\circ} \mathrm{C}\right)$ & std & $T_{\text {diss }}\left({ }^{\circ} \mathrm{C}\right)$ & std & $T_{e}\left({ }^{\circ} \mathrm{C}\right)$ & $\begin{array}{c}\text { Conc. } \\
\text { (g } \\
\text { Solvent } \\
\text { / g } \\
\text { pABA) }\end{array}$ & $\begin{array}{l}\text { cooling } \\
\text { rate } \\
\left({ }^{\circ} \mathrm{C} / \mathrm{min}\right)\end{array}$ & $T_{c}\left({ }^{\circ} \mathrm{C}\right)$ & std & $T_{\text {diss }}\left({ }^{\circ} \mathrm{C}\right)$ & std & $T_{e}\left({ }^{\circ} \mathrm{C}\right)$ \\
\hline \multirow[t]{4}{*}{0.06} & 0.3 & 13.2 & 2.4 & 23.1 & 0.1 & \multirow[t]{4}{*}{21.0} & \multirow[t]{4}{*}{0.06} & 0.3 & 30.7 & 1.6 & 41.2 & 0.2 & \multirow[t]{4}{*}{40.8} \\
\hline & 0.5 & 9.6 & 1.5 & 23.7 & 4.1 & & & 0.5 & 27.7 & 1.9 & 40.5 & 0.0 & \\
\hline & 0.7 & 8.0 & 0.0 & 25.7 & 0.1 & & & 0.7 & 26.5 & 0.7 & 42.2 & 0.2 & \\
\hline & 1.0 & 5.0 & 0.1 & 25.2 & 0.1 & & & 1.0 & 24.6 & 0.6 & 41.4 & 0.1 & \\
\hline \multirow[t]{4}{*}{0.09} & 0.3 & 27.5 & 2.4 & 34.2 & 0.1 & \multirow[t]{4}{*}{33.1} & \multirow[t]{4}{*}{0.07} & 0.3 & 33.7 & 1.7 & 44.8 & 0.1 & \multirow[t]{4}{*}{44.3} \\
\hline & 0.5 & 23.3 & 3.2 & 34.4 & 0.0 & & & 0.5 & 32.0 & 1.5 & 44.4 & 0.1 & \\
\hline & 0.7 & 21.0 & 1.9 & 36.6 & 0.1 & & & 0.7 & 31.0 & 1.2 & 45.6 & 0.1 & \\
\hline & 1.0 & 16.4 & 2.7 & 36.3 & 0.1 & & & 1.0 & 30.3 & 1.2 & 45.4 & 0.2 & \\
\hline \multirow[t]{3}{*}{0.12} & 0.3 & 34.1 & 2.2 & 42.4 & 0.1 & \multirow[t]{3}{*}{41.3} & \multirow[t]{3}{*}{0.08} & 0.3 & 38.1 & 1.7 & 47.8 & 0.1 & \multirow[t]{3}{*}{47.4} \\
\hline & 0.5 & 32.5 & 0.7 & 42.6 & 0.1 & & & 0.5 & 35.4 & 4.4 & 47.1 & 0.1 & \\
\hline & 0.7 & 31.0 & 1.4 & 45.4 & 0.3 & & & 0.7 & 33.2 & 2.5 & 48.6 & 0.1 & \\
\hline
\end{tabular}




\begin{tabular}{|c|c|c|c|c|c|c|c|c|c|c|c|c|c|}
\hline & 1.0 & 30.6 & 0.8 & 44.8 & 0.1 & & & 1.0 & 31.7 & 2.1 & 47.9 & 0.1 & \\
\hline \multirow[t]{4}{*}{0.15} & 0.3 & 42.8 & 0.9 & 51.2 & 0.1 & 50.1 & 0.09 & 0.3 & 41.0 & 1.3 & 51.1 & 0.2 & 50.6 \\
\hline & 0.5 & 38.5 & 0.4 & 51.5 & 0.2 & & & 0.5 & 40.0 & 0.8 & 50.9 & 0.1 & \\
\hline & 0.7 & 36.0 & 1.6 & 54.1 & 0.1 & & & 0.7 & 39.0 & 0.8 & 52.0 & 0.1 & \\
\hline & 1.0 & 33.1 & 1.4 & 53.7 & 0.1 & & & 1.0 & 37.8 & 1.2 & 51.9 & 0.1 & \\
\hline \multicolumn{7}{|c|}{ EtOH: $\mathrm{H}_{2} \mathrm{O} 2: 8$} & \multicolumn{7}{|c|}{ EtOH: $\mathrm{H}_{2} \mathrm{O} 1: 9$} \\
\hline $\begin{array}{l}\text { Conc. (g } \\
\text { Solvent / } \\
\text { g pABA) }\end{array}$ & $\begin{array}{l}\text { cooling } \\
\text { rate } \\
\left({ }^{\circ} \mathrm{C} / \mathrm{min}\right)\end{array}$ & $T_{c}\left({ }^{\circ} \mathrm{C}\right)$ & std & $T_{\text {diss }}\left({ }^{\circ} \mathrm{C}\right)$ & std & $T_{e}\left({ }^{\circ} \mathrm{C}\right)$ & $\begin{array}{c}\text { Conc. } \\
\text { (g } \\
\text { Solvent } \\
\text { / g } \\
\text { pABA) }\end{array}$ & $\begin{array}{l}\text { cooling } \\
\text { rate } \\
\left({ }^{\circ} \mathrm{C} / \mathrm{min}\right)\end{array}$ & $T_{c}\left({ }^{\circ} \mathrm{C}\right)$ & std & $T_{\text {diss }}\left({ }^{\circ} \mathrm{C}\right)$ & std & $T_{e}\left({ }^{\circ} \mathrm{C}\right)$ \\
\hline \multirow[t]{4}{*}{0.003} & 0.3 & 32.5 & 1.5 & 42.1 & 0.1 & 41.7 & 0.005 & 0.3 & 11.5 & 1.3 & 19.0 & 0.8 & 19.1 \\
\hline & 0.5 & 31.3 & 1.0 & 41.7 & 0.1 & & & 0.5 & 10.4 & 0.9 & 18.7 & 0.2 & \\
\hline & 0.7 & 31.0 & 1.5 & 42.8 & 0.1 & & & 0.7 & 9.6 & 1.1 & 18.7 & 0.2 & \\
\hline & 1.0 & 30.0 & 1.7 & 42.5 & 0.1 & & & 1.0 & 8.8 & 0.9 & 18.5 & 0.2 & \\
\hline \multirow[t]{4}{*}{0.004} & 0.3 & 36.2 & 1.9 & 47.0 & 0.3 & 46.7 & 0.01 & 0.3 & 27.0 & 0.3 & 33.9 & 0.3 & 33.8 \\
\hline & 0.5 & 34.5 & 1.4 & 46.2 & 0.2 & & & 0.5 & 25.5 & 1.4 & 33.2 & 0.2 & \\
\hline & 0.7 & 33.4 & 2.5 & 47.3 & 0.1 & & & 0.7 & 24.7 & 0.9 & 33.2 & 0.1 & \\
\hline & 1.0 & 31.4 & 1.7 & 46.8 & 0.1 & & & 1.0 & 23.7 & 1.2 & 33.3 & 0.3 & \\
\hline \multirow[t]{4}{*}{0.005} & 0.3 & 49.0 & 0.6 & 52.9 & 0.3 & 53.0 & 0.015 & 0.3 & 35.4 & 0.9 & 42.7 & 0.1 & 42.8 \\
\hline & 0.5 & 45.4 & 3.3 & 53.9 & 0.2 & & & 0.5 & 34.4 & 1.7 & 42.1 & 0.4 & \\
\hline & 0.7 & 44.5 & 2.0 & 53.2 & 0.2 & & & 0.7 & 33.4 & 1.2 & 41.9 & 0.2 & \\
\hline & 1.0 & 40.5 & 2.7 & 53.5 & 0.1 & & & 1.0 & 32.5 & 0.9 & 41.8 & 0.1 & \\
\hline \multirow[t]{4}{*}{0.006} & 0.3 & 49.1 & 1.3 & 56.3 & 0.2 & 56.1 & 0.002 & 0.3 & 43.3 & 0.7 & 48.8 & 0.0 & 49.2 \\
\hline & 0.5 & 47.2 & 0.8 & 56.0 & 0.1 & & & 0.5 & 40.4 & 1.1 & 48.6 & 0.2 & \\
\hline & 0.7 & 46.3 & 1.9 & 56.9 & 0.1 & & & 0.7 & 39.4 & 0.5 & 48.3 & 1.0 & \\
\hline & 1.0 & 44.4 & 0.6 & 56.3 & 0.3 & & & 1.0 & 38.3 & 0.5 & 48.0 & 0.2 & \\
\hline & & & $\mathrm{H}_{2} \mathrm{O}$ & & & & & & & & & & \\
\hline
\end{tabular}




\begin{tabular}{|ccccccc|}
\cline { 1 - 3 } $\begin{array}{c}\text { Conc. }(\mathrm{g} \\
\text { Solvent } /\end{array}$ & $\begin{array}{c}\text { cooling } \\
\text { rate } \\
\text { g pABA })\end{array}$ & $T_{c}\left({ }^{\circ} \mathrm{C}\right)$ & std & $T_{\text {diss }}\left({ }^{\circ} \mathrm{C}\right)$ & std & $T_{e}\left({ }^{\circ} \mathrm{C}\right)$ \\
\hline 0.005 & 0.3 & 18.0 & 0.6 & 28.3 & 0.3 & 27.4 \\
& 0.5 & 17.0 & 0.7 & 28.0 & 0.1 & \\
& 0.7 & 16.5 & 0.5 & 29.4 & 1.0 & \\
& 1.0 & 16.0 & 0.3 & 29.5 & 0.6 & \\
\hline 0.007 & 0.3 & 27.6 & 0.7 & 35.3 & 0.9 & 35.1 \\
& 0.5 & 27.0 & 0.9 & 36.3 & 0.6 & \\
& 0.7 & 26.4 & 0.9 & 36.0 & 0.4 & \\
& 1.0 & 25.7 & 0.5 & 36.7 & 0.6 & \\
\hline 0.009 & 0.3 & 34.5 & 0.8 & 42.3 & 0.6 & 42.0 \\
& 0.5 & 33.4 & 0.8 & 42.1 & 0.4 & \\
& 0.7 & 32.9 & 0.8 & 41.5 & 1.1 & \\
\hline 0.011 & 1.0 & 32.1 & 0.7 & 42.5 & 1.0 & \\
& 0.3 & 41.4 & 2.1 & 48.0 & 0.5 & 48.0 \\
& 0.5 & 40.6 & 1.8 & 48.1 & 0.7 & \\
& 0.7 & 39.5 & 0.7 & 46.6 & 0.2 & \\
& 1.0 & 38.5 & 0.6 & 47.9 & 0.3 & \\
\hline
\end{tabular}




\section{S6. Antisolvent Temperature Calibrations}

Figure S6 display antisolvent addition temperature calibrations performed for different initial solution volumes at different antisolvent addition temperatures. The increased effect antisolvent temperature has when more is added can be seen by the slopes of the linear regressions plotted.
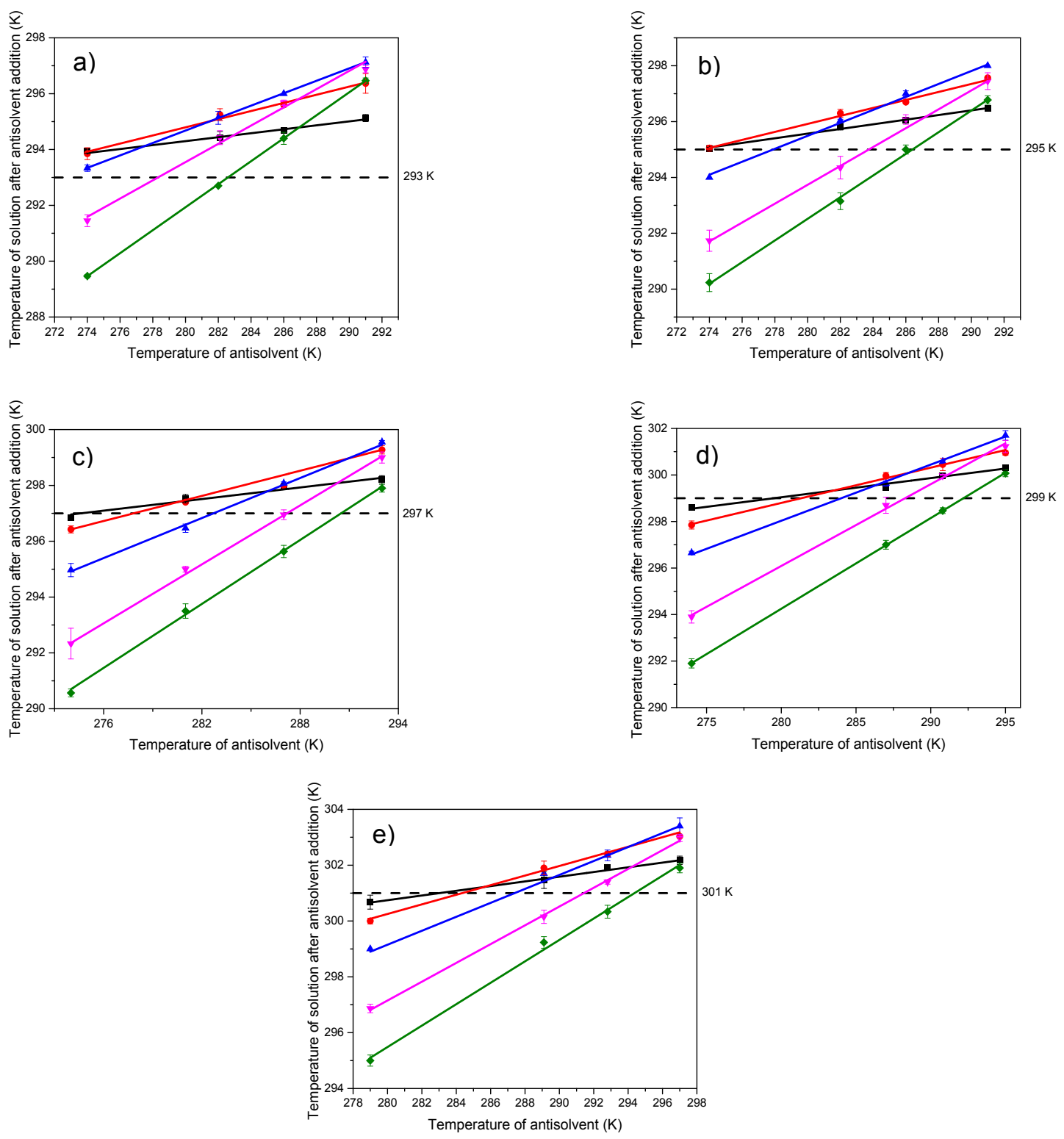

Figure S6. Effect antisolvent temperature had on solutions of pABA in $\mathrm{EtOH}: \mathrm{H} 2 \mathrm{O}$ mixed solvent held at constant temperature. Effect of antisolvent temperature on 0.2 $\mathrm{ml}$ initial solution $(\diamond), 0.3 \mathrm{ml}$ initial solution $(\nabla), 0.5 \mathrm{ml}$ initial solution $(\Delta), 0.65 \mathrm{ml}$ initial solution $(\bullet)$ and $0.8 \mathrm{ml}$ initial solution $(\boldsymbol{\bullet})$ shown by the gradients of the linear relationships. a) $293 \mathrm{~K}$, b) $295 \mathrm{~K}$, c) $297 \mathrm{~K}$, d) $299 \mathrm{~K}$, e) $301 \mathrm{~K}$. 


\section{S7. Isothermal analysis}

Figure S7 displays plots used to determine $\gamma_{\text {eff }}$ at each individual composition used, over the entire range of experimental temperatures. The slopes of the linear regressions, equal to $\frac{16 \pi v_{0}^{2} \gamma_{e f f}^{3}}{3(1+m d) k^{3}}$, were used to calculate $\gamma_{e f f}$.
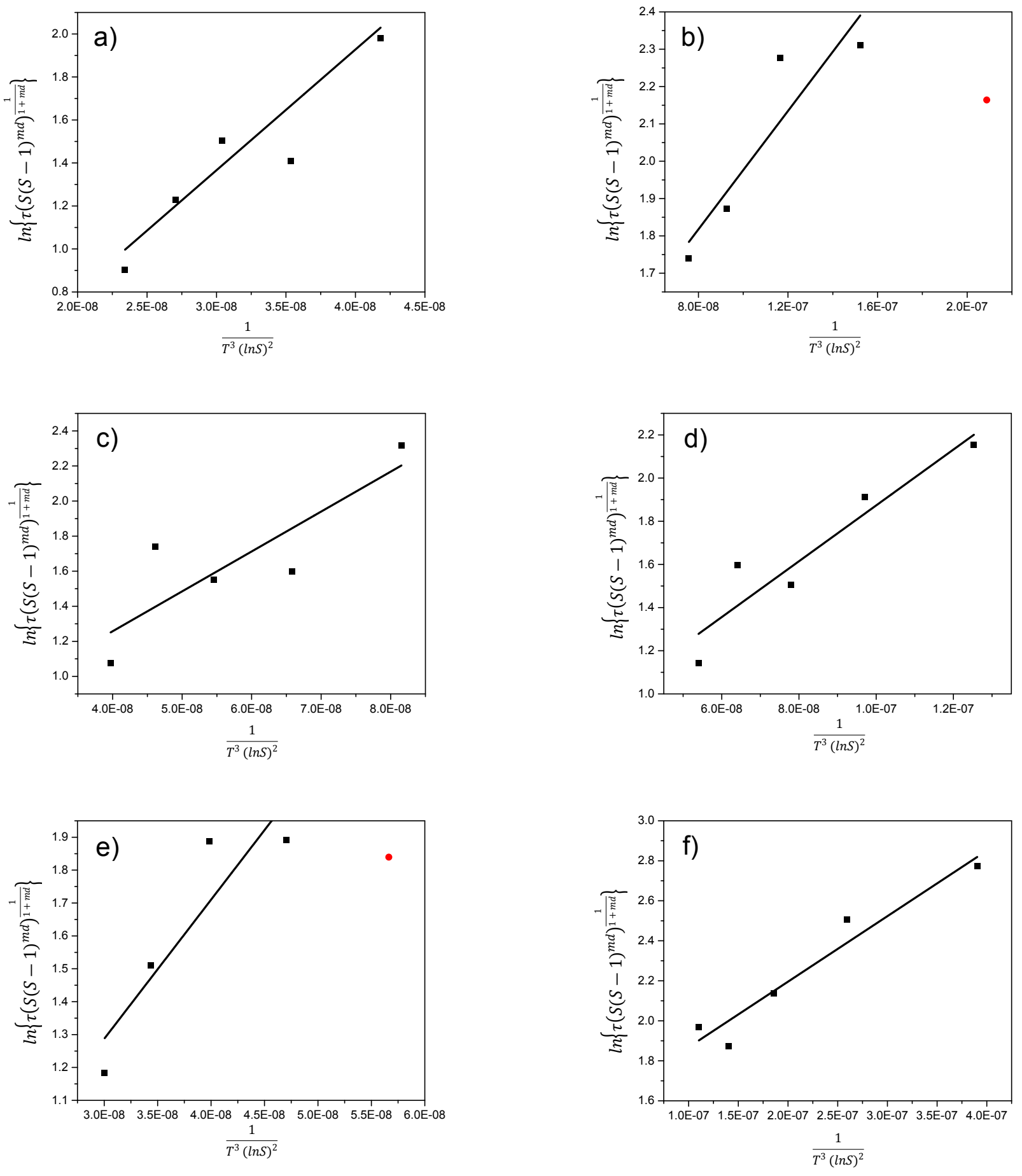

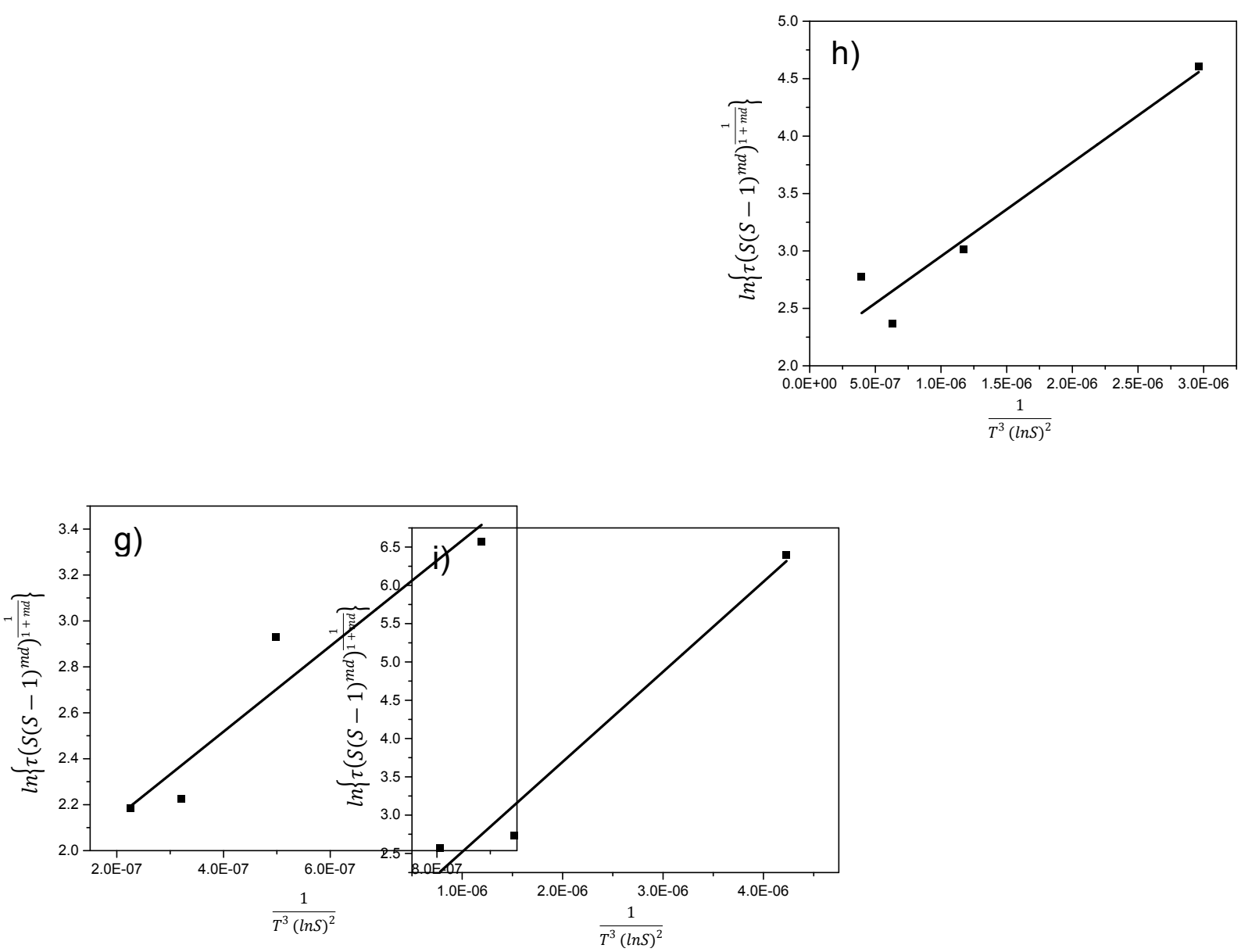

Figure S7. Induction time data to determine linear slope values for calculation of $\gamma_{\text {eff }}$ from data obtained. a) $34.4 \mathrm{~g} \mathrm{pABA} \mathrm{kg}^{-1}$ solvent mixture, b) $40.7 \mathrm{~g} \mathrm{pABA} \mathrm{kg}^{-1}$ solvent mixture, c) $47.0 \mathrm{~g} \mathrm{pABA} \mathrm{kg}^{-1}$ solvent mixture, d) $53.6 \mathrm{~g} \mathrm{pABA} \mathrm{kg}^{-1}$ solvent mixture, e) $60.3 \mathrm{~g} \mathrm{pABA} \mathrm{kg}^{-1}$ solvent mixture, f) $67.2 \mathrm{~g} \mathrm{pABA} \mathrm{kg}^{-1}$ solvent mixture, g) $74.2 \mathrm{~g}$ pABA $\mathrm{kg}^{-1}$ solvent mixture, h) $81.4 \mathrm{~g} \mathrm{pABA} \mathrm{kg}^{-1}$ solvent mixture, i) $88.9 \mathrm{~g} \mathrm{pABA} \mathrm{kg}^{-1}$ solvent mixture. Red circles in a) and d) represent outlier data not used in $\gamma_{e f f}$ calculation. 


\section{S8. Nucleation mechanism change}

Plots to determine whether a mechanistic change from HEN to HON over the supersaturation range used for each experimental temperature used are displayed in figure S8.
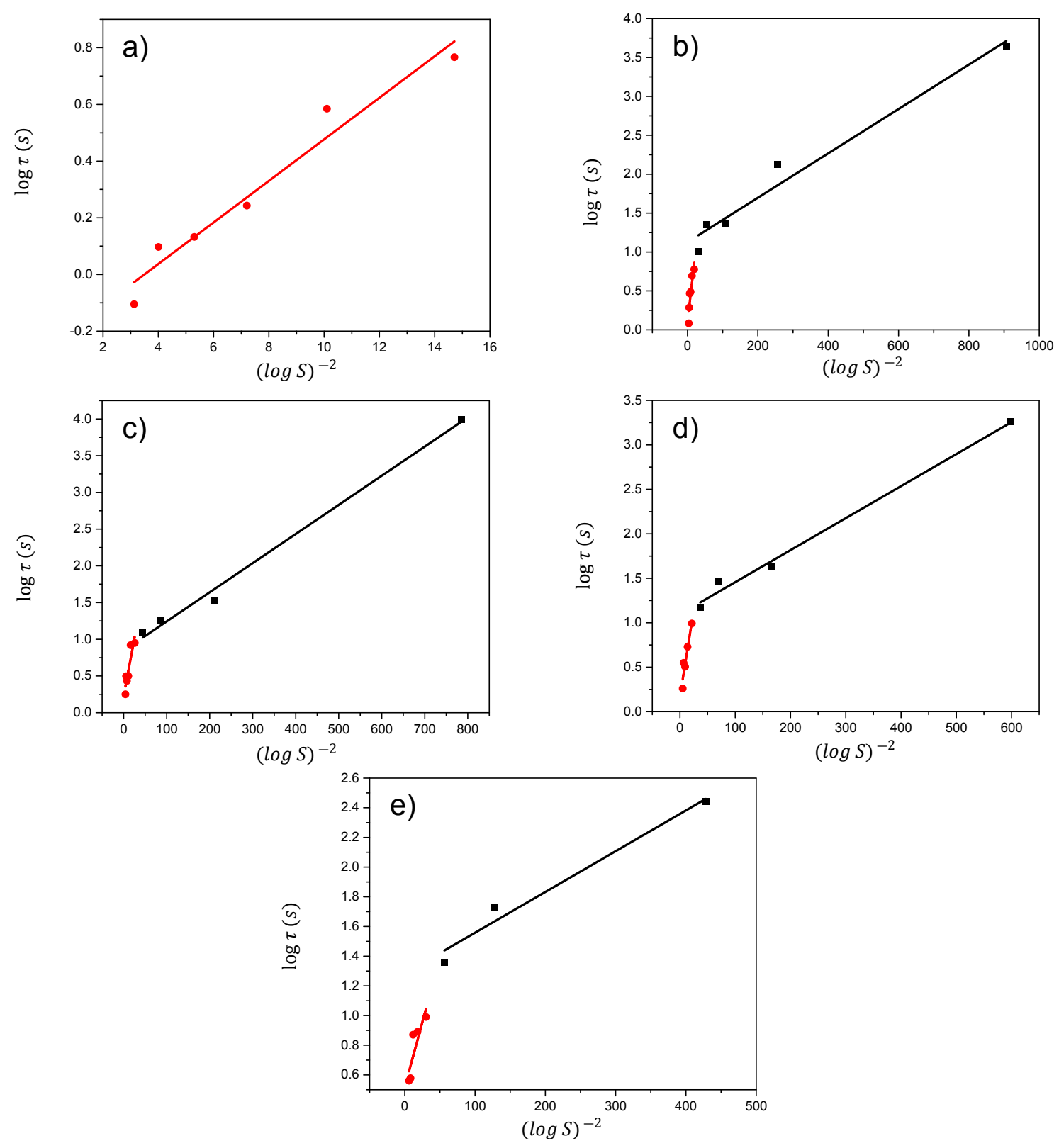

Figure S8. Induction time vs. supersaturation for single temperature and multiple composition solutions of pABA in EtOH: $\mathrm{H}_{2} \mathrm{O}$ mixtures. a) $293 \mathrm{~K}$, b) $295 \mathrm{~K}$, c) $297 \mathrm{~K}$, d) $299 \mathrm{~K}$, e) $301 \mathrm{~K} .(\bullet)$ region of homogeneous nucleation. (घ) region of heterogeneous nucleation. 
The finding of a nucleation mechanism change is of significant importance, especially in industry, as the mechanism by which nucleation takes place can have a large impact on the product formed under operation and as such, different operating conditions could mean that there is a large change in batch-to-catch variance of products, effecting final product quality.

Industrial crystallisations are usually performed in the HEN region of crystallisation, with encrustation being a large negative outcome of large scale operation ${ }^{7-9}$. If it is known that a process could be run in the HON region, then these issues could be overcome. 


\section{S8. Critical nucleus}

Table S2 displays the values of $r^{*}$ and $i^{*}$ calculated under the IbD methodology for all compositions studied. Initial Volume represents the initial solution volume present in the vial before antisolvent addition to make volume up to $1 \mathrm{ml}$.

\begin{tabular}{|c|c|c|c|c|c|c|c|}
\hline $\begin{array}{c}\text { Initial } \\
\text { Volume } \\
\text { (ml) }\end{array}$ & $\boldsymbol{S}$ & $\begin{array}{c}\boldsymbol{r}^{*} \\
\mathbf{( n m})\end{array}$ & $\begin{array}{c}\boldsymbol{i}^{*} \\
\text { (molecules) }\end{array}$ & $\begin{array}{c}\text { Initial } \\
\text { Volume } \\
\text { (m) }\end{array}$ & $\boldsymbol{S}$ & $\begin{array}{c}\boldsymbol{r}^{*} \\
\text { (nm) }\end{array}$ & $\begin{array}{c}\boldsymbol{i}^{*} \\
\text { (molecules) }\end{array}$ \\
\hline $\mathbf{0 . 3}$ & 3.68 & 0.53 & 3.70 & $\mathbf{0 . 3 5}$ & 3.16 & 0.56 & 4.42 \\
$\mathbf{0 . 3}$ & 3.36 & 0.56 & 4.50 & $\mathbf{0 . 3 5}$ & 2.90 & 0.60 & 5.45 \\
$\mathbf{0 . 3}$ & 3.07 & 0.60 & 5.58 & $\mathbf{0 . 3 5}$ & 2.66 & 0.65 & 6.88 \\
$\mathbf{0 . 3}$ & 2.80 & 0.65 & 7.07 & $\mathbf{0 . 3 5}$ & 2.44 & 0.71 & 8.91 \\
$\mathbf{0 . 3}$ & 2.55 & 0.71 & 9.18 & $\mathbf{0 . 3 5}$ & 2.24 & 0.78 & 11.89 \\
\hline $\mathbf{0 . 4}$ & 2.72 & 0.52 & 3.61 & $\mathbf{0 . 4 5}$ & 2.36 & 0.50 & 3.25 \\
$\mathbf{0 . 4}$ & 2.51 & 0.57 & 4.57 & $\mathbf{0 . 4 5}$ & 2.18 & 0.55 & 4.25 \\
$\mathbf{0 . 4}$ & 2.31 & 0.62 & 5.93 & $\mathbf{0 . 4 5}$ & 2.01 & 0.61 & 5.75 \\
$\mathbf{0 . 4}$ & 2.12 & 0.68 & 7.94 & $\mathbf{0 . 4 5}$ & 1.86 & 0.68 & 8.09 \\
$\mathbf{0 . 4}$ & 1.96 & 0.76 & 11.05 & $\mathbf{0 . 4 5}$ & 1.72 & 0.78 & 11.97 \\
\hline $\mathbf{0 . 5}$ & 2.06 & 0.51 & 3.31 & $\mathbf{0 . 5 5}$ & 1.82 & 0.46 & 2.40 \\
$\mathbf{0 . 5}$ & 1.91 & 0.56 & 4.53 & $\mathbf{0 . 5 5}$ & 1.69 & 0.52 & 3.48 \\
$\mathbf{0 . 5}$ & 1.77 & 0.63 & 6.46 & $\mathbf{0 . 5 5}$ & 1.57 & 0.60 & 5.35 \\
$\mathbf{0 . 5}$ & 1.64 & 0.73 & 9.73 & $\mathbf{0 . 5 5}$ & 1.46 & 0.71 & 8.92 \\
$\mathbf{0 . 5}$ & 1.52 & 0.85 & 15.77 & $\mathbf{0 . 5 5}$ & 1.36 & 0.87 & 16.66 \\
\hline $\mathbf{0 . 6}$ & 1.51 & 0.54 & 4.04 & $\mathbf{0 . 6 5}$ & 1.37 & 0.55 & 4.11 \\
$\mathbf{0 . 6}$ & 1.41 & 0.65 & 6.93 & $\mathbf{0 . 6 5}$ & 1.28 & 0.69 & 8.33 \\
$\mathbf{0 . 6}$ & 1.32 & 0.81 & 13.49 & $\mathbf{0 . 6 5}$ & 1.20 & 0.95 & 21.37 \\
$\mathbf{0 . 6}$ & 1.23 & 1.08 & 32.25 & $\mathbf{0 . 6 5}$ & 1.12 & 1.51 & 86.91 \\
\hline $\mathbf{0 . 7}$ & 1.25 & 0.84 & 15.20 & & & & \\
$\mathbf{0 . 7}$ & 1.17 & 1.18 & 41.55 & & & & \\
$\mathbf{0 . 7}$ & 1.10 & 1.98 & 195.46 & & & & \\
\hline & & & & & & &
\end{tabular}




\section{References}

(1) Camacho Corzo, D. .; Ma, C. .; Ramachandran, V.; Mahmud, T.; Roberts, K. . Crystallisation Route Map. In Engineering Crystallography: From Molecule to Crystal to Functional Form; Roberts, K. ., Docherty, R., Tamura, R., Eds.; Springer Science + Business Media, 2017; pp 179-213.

(2) Kashchiev, D. Nucleation; Butterworth-Heinemann: Oxford, 2000.

(3) Kashchiev, D.; Borissova, A.; Hammond, R. B.; Roberts, K. J. Dependence of the Critical Undercooling for Crystallization on the Cooling Rate. Journal of Physical Chemistry B 2010, $114 \quad$ (16), 5441-5446. https://doi.org/10.1021/jp100202m.

(4) Kashchiev, D.; Borissova, A.; Hammond, R. B.; Roberts, K. J. Effect of Cooling Rate on the Critical Undercooling for Crystallization. Journal of Crystal Growth 2010, 312 (5), 698-704. https://doi.org/10.1016/j.jcrysgro.2009.12.031.

(5) Mullin, J. W. Crystallization, 4th ed.; Butterworth-Heinemann: Oxford, 2001.

(6) Technobis. Crystal16 https://www.crystallizationsystems.com/crystal16.

(7) Liang, K.; White, G.; Wilkinson, D.; Ford, L. J.; Roberts, K. J.; Wood, W. M. L. An Examination into the Effect of Stirrer Material and Agitation Rate on the Nucleation of L-Glutamic Acid Batch Crystallized from Supersaturated Aqueous Solutions. Crystal Growth and Design 2004, 4 (5), 1039-1044.

(8) Liang, K.; White, G.; Wilkinson, D.; Ford, L. J.; Roberts, K. J.; Wood, W. M. L. Examination of the Process Scale Dependence of I -Glutamic Acid Batch Crystallized from Supersaturated Aqueous Solutions in Relation to Reactor Hydrodynamics. Industrial \& Engineering Chemistry Research 2004, 43 (5), 1227-1234.

(9) Al-Marri, M.; Mahmud, T.; Roberts, K. J. A Flexible Heat Transfer Model for Simulating the Performance of Batch Cooling Crystallization as a Function of Process Scale Size. In Proceedings of the 17th International Symposium on Industrial Crystallization; Ulrich, J., Jansens, P., Roelands, M., Eds.; Vol. 2, 2008; pp 613-620. 